\title{
Coronary interventions via radial artery without pre-procedural routine use of spasmolytic agents
}

\author{
Tomasz Bochenek ${ }^{1}$, Michał Lelek ${ }^{1}$, Małgorzata Kowal-Kałamajka ${ }^{1}$, Błażej Kusz ${ }^{1}$, Jan Szczogiel ${ }^{1}$, \\ Andrzej Jaklik ${ }^{1}$, Tomasz Roleder ${ }^{2}$, Katarzyna Mizia-Stec ${ }^{1}$
}

${ }^{1}$ First Department of Cardiology, Medical University of Silesia, Katowice, Poland

${ }^{2}$ Regional Specialist Hospital, Research and Development Center, Wroclaw, Poland

Adv Interv Cardiol 2020; 16, 2 (60): 138-144

DOI: https://doi.org/10.5114/aic.2020.96056

\begin{abstract}
Introduction: Radial access reduces the number of vascular complications. Radial artery spasm (RAS) can be prevented by the use of spasmolytic agents. However, use of these drugs can be possibly limited to certain groups of patients.

Aim: To assess the feasibility and safety of coronary angiography and percutaneous coronary interventions through the radial artery without the routine use of spasmolytic agents.

Material and methods: A group of 293 patients (M/F 180/113, mean age: $67 \pm 10$ years) who underwent coronary angiography and interventions through the radial artery approach was studied. Spasmolytic agents were applied in case of RAS. Every patient had ultrasound assessment of the radial artery on the next day to assess its diameter and detect occlusion.

Results: RAS was observed in 55 patients (18.8\%, M/F 28/27) and radial artery occlusion (RAO) in 47 (16\%, M/F: 24/23) cases. RAS was followed by RAO in 17 cases, which constituted 17/55 (30.9\%) of all RAS. Two patients had symptomatic occlusion, which required prolonged anticoagulation with complete restoration of patency. The RAS was higher in prolonged procedures (angiography time $32.6 \pm 12.8$ vs. $29 \pm 13.5 \mathrm{~min}, p=0.03$; intervention time $40 \pm 23.5$ vs. $26.3 \pm 25 \mathrm{~min}, p=0.0035)$ and was dependent on time of the local pressure ( $7.5 \pm 2.3$ vs. $6.5 \pm 2.8 \mathrm{~h}, p=0.03$ ). The RAO increased proportionally to the number of catheters used $(p=0.01)$ and was dependent on time of the local pressure ( $8.6 \pm 3.5$ vs. $6.4 \pm 2.7 \mathrm{~h}, p<0.001)$.

Conclusions: Our study showed that angiography and interventions without routine use of spasmolytic agents were feasible and safe. RAS and RAO are related to independent risk factors and comparable to data from the literature when spasmolytics were used.
\end{abstract}

Key words: percutaneous, occlusion, spasm.

S u m m a ry

Until now coronary interventions via the radial artery are usually performed with routine use of spasmolytics. The major finding of our study is that the rate of coronary spasms and occlusions is comparable to rates when those agents are used. Routine use of spasmolytics with evolution of new devices might be limited to selected groups of patients.

\section{Introduction}

Radial access dominated coronary interventions across the world and significantly reduced the number of vascular complications [1]. The small number of the observed radial access complications correlates with the experience of the operator and is also related to the type of the access device [2]. The most common complication is the radial artery spasm (RAS) and radial artery occlusion (RAO) [3]. It can be prevented by the appropriate choice of vascular access and minimization of the equipment use.
The use of spasmolytic agents has a strong relation to avoiding vascular access complications. Previous data documented efficacy of various types of vasodilators in preventing radial artery spasm [4-6]. However, with the fast evolution of current devices and the improvement of their flexibility, dimension, deliverability and quality of materials the routine use of spasmolytics should be constantly validated and can be possibly limited only to certain pre-selected groups of patients.

Previous studies analyzed whether use of spasmolytics has a potential to decrease the complications of radial ac-

\section{Corresponding author:}

Tomasz Bochenek MD, PhD, First Department of Cardiology, Medical University of Silesia, 47 Ziolowa St, 40-635 Katowice, Poland, phone: +48 660689 250, e-mail: tbochun1@gmail.com

Received: 18.11.2019, accepted: 20.01.2020. 
cess [7] However, the question arises whether the use of spasmolytic is really necessary during the radial access. The application of vasodilators may not be appropriate in patients with myocardial infarction and low blood pressure, who will benefit from radial access the most. Although the initial results of spasmolytic avoidance are promising, there are just a few reports presenting the safety of the radial access without the application of spasmolytics.

\section{Aim}

Therefore, the aim of our study is to assess the feasibility and safety of coronary angiography and percutaneous coronary interventions performed through the radial artery without the routine use of spasmolytic agents.

\section{Material and methods}

We conducted a single-center retrospective study. Data were obtained in the First Department of Cardiology of the Upper Silesian Medical Center and represented the period between October 2016 and January 2018. In this period all consecutive patients in whom angiography via the radial artery without routine use of spasmolytic agents was performed were enrolled in the study. It was an all-comers study. The selection of patients was independent from any inclusion or exclusion criteria, and no pre-selection protocol was used. All operators had already stopped using routinely spasmolytic in the radial approach based on their own observations. We analyzed $13 \%$ of patients treated via the radial artery in our department in this period (Figure 1).

\section{Radial access technique}

All the patients had radial artery pulse palpation without the Allen test performed before the procedure.

All the radial procedures were performed through the right radial artery. None of the patients received premedication. Only $2 \mathrm{ml}$ of lidocaine was injected subcutaneously before the radial artery puncture to adequately anesthetize the puncture site. The radial artery access was achieved by the use of open bore needles, 0.025 " hydrophilic short guidewires and 6 Fr or 5 Fr Balton vascular access sheaths. After the sheath insertion $5000 \mathrm{IU}$ of unfractionated heparin was injected as a bolus in order to minimize the chance of radial artery occlusion.

In all the patients $0.035^{\prime \prime}$ guidewires were used for advancing the catheters and standard $5 \mathrm{Fr}$ or $6 \mathrm{Fr}$ catheters in all the procedures. Immediately after the procedure the vascular sheath was removed and standard pressure compression bands that were inflated with air until bleeding subsided were used to achieve full hemostasis of the access site.

Spasmolytic agents were only applied during the radial artery spasm. Papaverine, nitrates or verapamil in standard doses were used for that purpose.

\section{Periprocedural assessment}

After the pressure band was removed when bleeding resolved completely, not earlier than $3 \mathrm{~h}$ after the procedure, every patient had ultrasound assessment of the radial and brachial artery on the next day to assess its maximal and minimal diameter or to detect its occlusion. Every imaging of the radial artery was performed post-procedurally, in cross sectional imaging, by placing the probe $2 \mathrm{~cm}$ proximal to the styloid process of the radius, perpendicular to the vessel wall - above the access point. Moreover, every patient was asked to describe pain intensity during the radial procedure on a ten-point scale. Ten points represented the maximal pain intensity and zero corresponded to no pain at all. Occlusion was defined as lack of flow in the radial artery seen in doppler ultrasonography and it was confirmed by simple palpation of the pulse at least $24 \mathrm{~h}$ after band removal.

\section{Statistical analysis}

Statistical analysis was performed using Statistica 10.0 (StatSoft Poland) software. Continuous variables were presented as mean \pm SD and categorical as absolute counts and percentages or as medians with interquartile range (IQR). The type of distribution was verified using the Shapiro-Wilk test. Relationships between variables were checked using the Mann-Whitney $U$ test, $\chi^{2}$ test and analysis of variance (ANOVA). Pearson's correlation coefficient was used to measure correlations between categorical variables. A $p$-value of less than 0.05 was considered statistically significant.

\section{Results}

\section{Clinical characteristics}

Overall 293 patients (M/F 180/113, mean age: $67 \pm 10$ ) who underwent coronary angiography and coronary interventions through the radial artery approach were analyzed.

Both patients with elective procedures (85/29\%) and with urgent interventions (208/71\%) in acute coronary syndromes were enrolled in the analysis. Among 293 patients enrolled in the study, 124 (42\%) underwent percutaneous coronary intervention with implantation of stent(s).

The following concomitant diseases were present in the study group: systemic hypertension (78\%), diabetes mellitus type 2 (26\%), hyperlipidemia (57\%), and peripheral artery disease (8\%). History of previous radial approach was

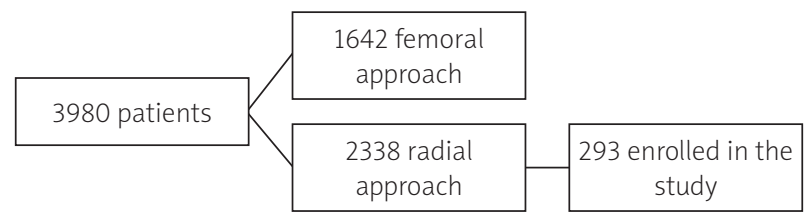

Figure 1. Flow chart of study 
present in $18 \%$ of all patients. Detailed characteristics of the study group are presented in Tables I and II. The local Bioethical Commission approved conduction of the study.

\section{Complications of radial artery access without spasmolytic agents}

Among the defined complications of radial artery access, RAS was observed in $55(18.8 \%)$ cases and RAO was diagnosed in $47(16 \%)$ cases. RAS was followed by RAO in 17 cases, which constituted 17 (55\%) of all RAS subjects. Only 2 patients had symptomatic occlusion, which required prolonged anticoagulation with complete restoration of patency.

\section{Catheters and radial artery size for RAS and RAO groups}

$6 \mathrm{Fr}$ catheters were used in 206 patients and $5 \mathrm{Fr}$ catheters were used in 87 patients. There was no difference in the mean diameter of the radial artery between $5 \mathrm{Fr}$ and $6 \mathrm{Fr}$ catheters used for coronary angiography (2.850 (IQR 2.60, 3.21) vs. 2.95 (IQR 2.65, 3.26), $p=0.325$ ). RAS occurred more frequently in $6 \mathrm{Fr}$ as compared to $5 \mathrm{Fr}$ catheters (45 (225) vs. 10 (12\%), $p=0.038)$, and RAO occurred more frequently in $6 \mathrm{Fr}(41(20 \%)$ vs. $6(7 \%)$, $p=0.0086)$.

Clinical characteristics of the study subgroups: RAS vs. no-RAS

The RAS and no-RAS subgroups did not differ in regard to most clinical parameters. History of coronary artery bypass grafting (CABG) was found more frequently in the RAS sub-group as compared to the no-RAS subgroup (13 vs. $4 \%, p=0.027$ ) (Table I).

Clinical characteristics of the study subgroups: RAO vs. no-RAO

The RAO and no-RAO subgroups did not differ in regard to most clinical parameters. RAO occurred in

Table I. Baseline characteristics of the study population and the subgroups RAS and no-RAS

\begin{tabular}{|c|c|c|c|c|}
\hline Parameter & Study population & RAS & No RAS & $P$-value \\
\hline Total number of patients & 293 & 55 & 238 & \\
\hline Men, $n(\%)$ & 180 & $28(51)$ & $152(64)$ & 0.078 \\
\hline Women, $n(\%)$ & 113 & $27(49)$ & $86(36)$ & \\
\hline Age [years] mean \pm SD & $67 \pm 10$ & $66 \pm 10$ & $67 \pm 10$ & 0.29 \\
\hline Height $[\mathrm{cm}]$ mean $\pm S D$ & $166 \pm 12.4$ & $165 \pm 17.5$ & $166 \pm 11$ & 0.991 \\
\hline Weight mean \pm SD & $83 \pm 17.1$ & $80 \pm 15.8$ & $84 \pm 17.3$ & 0.239 \\
\hline $\mathrm{BMI}\left[\mathrm{kg} / \mathrm{m}^{2}\right]$ mean $\pm \mathrm{SD}$ & $30 \pm 15$ & $32 \pm 23$ & $31 \pm 16$ & 0.273 \\
\hline History of CAD, $n(\%)$ & $188(64)$ & $35(64)$ & $153(64)$ & 0.94 \\
\hline \multicolumn{5}{|l|}{ Concomitant diseases, $n$ (\%): } \\
\hline DMT2 & $75(26)$ & $13(24)$ & $62(26)$ & 0.763 \\
\hline $\mathrm{HA}$ & $228(78)$ & $45(82)$ & $183(77)$ & 0.289 \\
\hline$\overline{P A D}$ & $24(8)$ & $5(9)$ & $19(80)$ & 0.761 \\
\hline$\overline{\mathrm{HL}}$ & $167(57)$ & $33(60)$ & $134(56)$ & 0.518 \\
\hline \multicolumn{5}{|l|}{ Smoking habits, $n(\%)$ : } \\
\hline History of smoking & $49(16)$ & $7(13)$ & $42(18)$ & 0.393 \\
\hline \multicolumn{5}{|l|}{ Case history data, $n$ (\%): } \\
\hline History of stroke & $17(6)$ & $3(6)$ & $14(6)$ & 0.926 \\
\hline History of MI & $84(29)$ & $20(36)$ & $64(27)$ & 0.175 \\
\hline $\begin{array}{l}\text { History of previous radial approach } \\
\text { coronarography }\end{array}$ & $53(18)$ & $13(24)$ & $40(17)$ & 0.248 \\
\hline History of $\mathrm{PCl}$ & $86(29)$ & 17 (31) & $69(29)$ & 0.779 \\
\hline History of CABG & $17(6)$ & $7(13)$ & $10(4)$ & 0.027 \\
\hline
\end{tabular}

RAS - radial artery spasm, BMI - body mass index, CAD - coronary artery disease, DMT2 - type 2 diabetes mellitus, HA - arterial hypertension, PAD - peripheral artery disease, $\mathrm{HL}$ - hyperlipidemia, $\mathrm{MI}$ - myocardial Infarction, $\mathrm{PCl}$ - percutaneous coronary interventions, CABG - coronary artery bypass graft. 
younger patients ( $64 \pm 10$ vs. $68 \pm 10, p=0.04$ ) while hyperlipidemia was found more often in no-RAO patients (Table II).

\section{Procedural and peri-procedural data: RAS vs. no-RAS}

The occurrence of RAS was significantly higher in prolonged procedures (coronary angiography time 32.6 \pm 12.8 vs. $29 \pm 13.5, p=0.03$; total time of coronary intervention time $40 \pm 23.5$ vs. $26.3 \pm 25, p=0.0035$ ) and increased proportionally to the number of catheters used $(p=0.08)$. It was also dependent on time of the local pressure (7.5 \pm 2.3 vs. $6.5 \pm 2.8, p=0.03)$ (Table III).

\section{Procedural and peri-procedural data: RAO vs. no-RAO}

The occurrence of RAO increased proportionally to the number of catheters used $(p=0.01)$ and was dependent on time of the local pressure $(8.6 \pm 3.5$ vs. $6.4 \pm 2.7)$ $(p=0.001)$ (Table IV, Figure 2).

\section{Regression analysis}

Occurrence of RAS was related to the following factors: CABG in case history $(R=0.181, p=0.027)$, percutaneous artery angioplasty in LCX $(R=0.263, p=0.001)$ or $\mathrm{D}$ artery $(R=0.207, p=0.011)$. There was also a positive correlation between the occurrence of RAS and the use of $6 \mathrm{~F}$ catheters ( $R=0.197, p=0.02$ ). Furthermore, the local pressure time correlated with the RAS occurrence $(R=0.244, p=0.003)$.

Occurrence of RAO correlated with the following factors: hyperlipidemia ( $R=0.181, p=0.028)$, CABG in case history $(R=0.203, p=0.01)$, in case of atherosclerotic changes $(R=0.72, p=0.001)$.

\section{Discussion}

The main reason for the increasing popularity of the radial approach is the simplicity of achieving effective hemostasis of the superficially running radial artery and reduction in the complications rate. Radial access

Table II. Baseline characteristics of the study subgroups RAO and no-RAO

\begin{tabular}{|c|c|c|c|}
\hline Parameter & RAO & No-RAO & $P$-value \\
\hline Total number of patients & 47 & 239 & - \\
\hline Men, $n(\%)$ & $24(51)$ & $156(65)$ & 0.11 \\
\hline Women, $n(\%)$ & $23(49)$ & $90(35)$ & \\
\hline Age [years] mean \pm SD & $64 \pm 10$ & $68 \pm 10$ & 0.04 \\
\hline Height $[\mathrm{cm}]$ mean \pm SD & $167 \pm 9.4$ & $166 \pm 12.8$ & 0.62 \\
\hline Weight, mean \pm SD & $81.9 \pm 14.7$ & $83.4 \pm 17.6$ & 0.51 \\
\hline $\mathrm{BMI}\left[\mathrm{kg} / \mathrm{m}^{2}\right]$ mean $\pm \mathrm{SD}$ & $29.4 \pm 4.9$ & $31.6 \pm 18.8$ & 0.54 \\
\hline History of CAD, $n(\%)$ & $30(64)$ & 158 & 0.9 \\
\hline \multicolumn{4}{|l|}{ Concomitant diseases, $n(\%)$ : } \\
\hline DMT2 N & $11(23)$ & $64(66)$ & 0.7 \\
\hline $\mathrm{HA}$ & $34(72)$ & $194(81)$ & 0.3 \\
\hline PAD & $3(6)$ & $21(9)$ & 0.6 \\
\hline $\mathrm{HL}$ & $20(43)$ & $147(62)$ & 0.027 \\
\hline \multicolumn{4}{|l|}{ Smoking habits, $n(\%)$ : } \\
\hline History of smoking & $9(19)$ & $40(17)$ & 0.64 \\
\hline \multicolumn{4}{|l|}{ Case history data, $n(\%)$ : } \\
\hline History of stroke & $1(2)$ & $16(7)$ & 0.19 \\
\hline History of MI & $16(34)$ & $68(28)$ & 0.39 \\
\hline History of previous radial approach coronarography & $9(19)$ & $44(18)$ & 0.84 \\
\hline History of $\mathrm{PCl}$ & $12(26)$ & $74(31)$ & 0.53 \\
\hline History of CABG & 0 & $17(7)$ & 0.01 \\
\hline
\end{tabular}

RAO - radial artery occlusion, BMI - body mass index, CAD - coronary artery disease, DMT2 - type 2 diabetes mellitus, HA - arterial hypertension, PAD - peripheral artery disease, $\mathrm{HL}$ - hyperlipidemia, $\mathrm{MI}$ - myocardial infarction, $\mathrm{PCl}$ - percutaneous coronary interventions, CABG - coronary artery bypass graft. 
Table III. Procedural and peri-procedural data: RAS vs. no-RAS subgroups

\begin{tabular}{|c|c|c|c|}
\hline Parameter & $\begin{array}{c}\text { RAS } \\
N(\%) \\
\text { Mean value } \pm \text { SD }\end{array}$ & $\begin{array}{c}\text { No-RAS } \\
N(\%) \\
\text { Mean value } \pm \text { SD }\end{array}$ & $P$-value \\
\hline Elective coronary procedure & $11(20 \%)$ & $74(31 \%)$ & 0.42 \\
\hline Coronary angiography & $28(51 \%)$ & $142(60 \%)$ & 0.24 \\
\hline Coronary angiography + coronary intervention & $27(49 \%)$ & $96(40 \%)$ & 0.24 \\
\hline Coronary angiography time [min] & $32.6 \pm 12.8$ & $29 \pm 13.5$ & 0.03 \\
\hline Coronary angioplasty time [min] including angiography & $40 \pm 23.5$ & $26.3 \pm 25$ & 0.0035 \\
\hline Pain level during procedure (points on 1-10 scale) & $3.8 \pm 2.5$ & $3.5 \pm 2.3$ & 0.45 \\
\hline \multicolumn{4}{|l|}{ Number of catheters used: } \\
\hline 1 catheter & $9(16 \%)$ & $52(22 \%)$ & 0.08 \\
\hline 2 catheters & $22(40 \%)$ & $116(49 \%)$ & \\
\hline 3 or more catheters & $24(44 \%)$ & $70(29 \%)$ & \\
\hline \multicolumn{4}{|l|}{ Number of stents used: } \\
\hline 1 stent & $11(20 \%)$ & $69(29 \%)$ & 0.14 \\
\hline 2 stents & $10(18 \%)$ & $20(8 \%)$ & \\
\hline 3 stents & $1(2 \%)$ & $5(2 \%)$ & \\
\hline \multicolumn{4}{|l|}{ Brachial artery measurements: } \\
\hline Brachial artery diameter [mm] & $5 \pm 0.6$ & $5.9 \pm 4.8$ & 0.147 \\
\hline Minimal brachial artery diameter [mm] & $3.5 \pm 3.9$ & $2.9 \pm 1.9$ & 0.443 \\
\hline Maximal brachial artery diameter [mm] & $3.2 \pm 0.5$ & $3.3 \pm 1.6$ & 0.697 \\
\hline Time of the local pressure $[\mathrm{h}]$ & $7.5 \pm 2.3$ & $6.5 \pm 2.8$ & 0.03 \\
\hline
\end{tabular}

RAS - radial artery spasm.

compared to femoral is more comfortable for patients, enables quick start-up after the procedure, and shortens the length of hospital stays. Furthermore, studies showed a significant decrease in the frequency of hemorrhagic complications in radial access [8]. In a randomized study, the incidence of arterial contraction was lower through the arterial administration of nitroglycerine or nitroglycerine combined with verapamil [4]. However our study proved that transradial coronary angiography and revascularization is both safe and feasible without routine initial use of spasmolytic agents.

In our study we had to make conversion to a femoral approach because of severe spasm only in $4.5 \%$ of patients of 293 patients enrolled in the study. The rate of access conversions remained at the same level irrespective of the use of spasmolytic agents. Our data are in line with the rate of conversion observed in routine application of spasmolytic agents. The metanalysis of 23 randomized studies comparing radial with femoral access in diagnostic and therapeutic coronary procedures mainly with spasmolytic agents reported a trans-radial approach failure rate of $5.9 \%$ [9].
Our study showed that the spasm and occlusion rate did not differ in regard to sex. This finding is contrary to a previous observation that women tend to have radial artery spasm more often. It was shown in our study that radial artery occlusion happened more often in younger patients. One possible explanation of the mechanism is that younger patients took periprocedurally smaller amounts of anticoagulation and antiplatelet agents because of fewer comorbidities.

History of CABG was found more frequently in the RAS. To perform coronary artery angiography in patients after CABG, the left radial access is required. Since operators are more familiar with right radial artery access, the left might have required a large number of attempts and thus provoke spasm. Unfortunately, we did not perform such thorough analysis and cannot prove this speculation. Furthermore, more frequent catheter changes are required to perform coronary angiography after CABG and longer procedural time might have also provoked RAS. Larger devices and their quantity may according to optical coherence tomography study lead to microdissections at the radial access site and also be 
Table IV. Procedural and peri-procedural data: RAO vs. no-RAO subgroups

\begin{tabular}{|c|c|c|c|}
\hline Parameter & $\begin{array}{c}\text { RAO } \\
N(\%) \\
\text { Mean value } \pm \text { SD }\end{array}$ & $\begin{array}{c}\text { No-RAO } \\
N(\%) \\
\text { Mean value } \pm \text { SD }\end{array}$ & $P$-value \\
\hline Elective coronary procedure & $8(17 \%)$ & 77 (32\%) & 0.037 \\
\hline Coronary angiography & $27(57 \%)$ & $136(57 \%)$ & 0.95 \\
\hline Coronary angiography + coronary intervention & $20(43 \%)$ & $103(43 \%)$ & 0.95 \\
\hline Coronary angiography time [min] & $27 \pm 13.5$ & $30 \pm 13.4$ & 0.19 \\
\hline Coronary angioplasty time [min] - including angiography & $25 \pm 23$ & $29.6 \pm 26$ & 0.43 \\
\hline Pain level during procedure (points on 1-10 scale) & $3.9 \pm 2.5$ & $3.6 \pm 2.3$ & 0.37 \\
\hline \multicolumn{4}{|l|}{ Number of catheters used: } \\
\hline 1 catheter & $4(9 \%)$ & $57(23 \%)$ & 0.01 \\
\hline 2 catheters & $21(45 \%)$ & $117(48 \%)$ & \\
\hline 3 or more catheters & $22(47 \%)$ & $72(29 \%)$ & \\
\hline \multicolumn{4}{|l|}{ Number of stents used: } \\
\hline 1 stent & $13(28 \%)$ & $67(27 \%)$ & 0.98 \\
\hline 2 stents & $5(11 \%)$ & $25(10 \%)$ & \\
\hline 3 stents & $1(2 \%)$ & $5(2 \%)$ & \\
\hline \multicolumn{4}{|l|}{ Brachial artery measurements: } \\
\hline Brachial artery diameter [mm] & $5.2 \pm 0.6$ & $5.5 \pm 4.6$ & 0.7 \\
\hline Minimal brachial artery diameter [mm] & $3.37 \pm 3.6$ & $2.98 \pm 2.2$ & 0.54 \\
\hline Maximal brachial artery diameter [mm] & $3.26 \pm 0.6$ & $3.28 \pm 1.5$ & 0.45 \\
\hline Time of the local pressure [h] & $8.6 \pm 3.5$ & $6.4 \pm 2.7$ & 0.001 \\
\hline
\end{tabular}

$R A O$ - radial artery occlusion.

a potent risk factor for radial artery occlusion [10]. As observed in our study, the presence of ultrasonographic evidence of atherosclerosis in the radial artery was more frequent among those who developed spasm or arterial occlusions. Radial arteries with atherosclerosis are more prone to damage and occlusion because of aforementioned dissection. It may be speculated that prolonged pressure promotes thrombus formation and thus results in total artery occlusion. Our finding is in line with previous studies showing that prolonged hemostasis was an independent predictor of radial artery occlusion [11]. Achieving good hemostasis without prolonged compression is a challenge after radial artery procedures. Probably novel, more advanced devices will help to solve this issue.

It was not a randomized study, but an all-comers one without a control group of patients, to whom spasmolytics were not administered during the procedure.

\section{Conclusions}

Our study showed that angiography and interventions performed through the radial artery without rou- tine use of spasmolytic agents seem to be feasible and relatively safe. In this approach the occurrence of RAS and/or RAO is related to standard independent risk factors and comparable to data from the literature when spasmolytics were used. Thus, their routine application

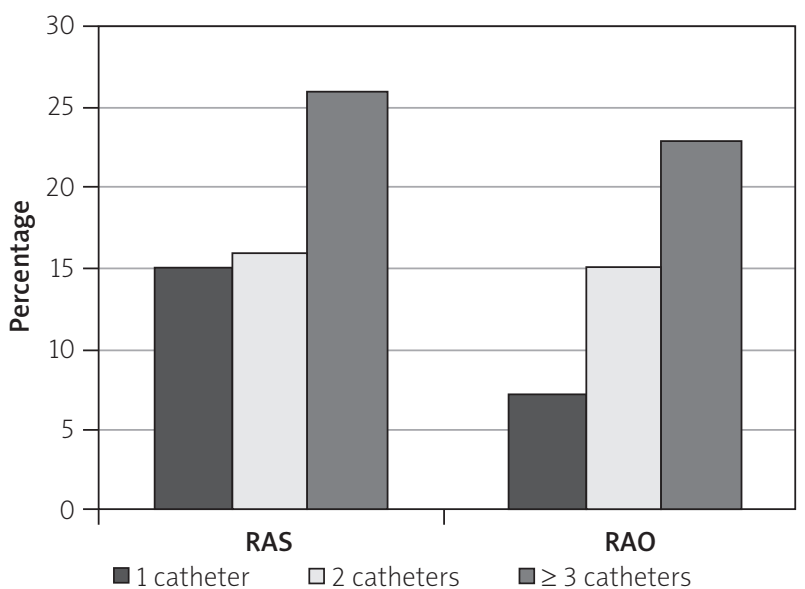

Figure 2. Occurrence of RAS and RAO depending on number of catheters and stents used 
may be significantly limited or even avoided on a daily clinical basis in selected patients.

\section{Conflict of interest}

The authors declare no conflict of interest.

\section{References}

1. Jolly SS, Yusuf S, Cairns J, et al. Radial versus femoral access for coronary angiography andintervention in patients with acute coronary syndromes (RIVAL): a randomised, parallel group, multicentre trial. Lancet 2011; 377: 1409-20.

2. Alkhouli M, Cohen HA, Bashir R. Radial artery avulsion - a rare complication of transradial catheterization. Catheter Cardiovasc Interv 2015; 85: E32-4.

3. Avdikos G, Karatasakis A, Tsoumeleas A, et al. Radial artery occlusion after transradial coronary catheterization. Cardiovasc Diagn Ther 2017; 7: 305-16.

4. Chen CW, Lin Cl, Lin T, et al. A simple and effective regimen for prevention of radial artery spasm during coronary catheterization. Cardiology 2006; 105: 43-7.

5. Varenne O, Jegou A, Cohen R, et al. Prevention of arteria spasm during percutaneous coronary interventions through radial artery. The Spam study. Catheter Cardiovasc Interv 2006; 68: 231-5.

6. Ruiz-Salmeron RJ, Mora R, Masotti M, Betriu A. Assessment of the efficacy of phentolamine to prevent radial artery spasm during cardiac catheterization procedures. Catheter Cardiovasc Interv 2005; 66: 192-8.

7. Kim SH, Kim EJ, Cheon WS, et al. Comparative study of nicorandil and a spasmolytic cocktail in preventing radial artery spasm during transradial coronary angiography. Int J Cardiol 2007; 120: 325-30.

8. Agostoni P, Biondi-Zoccai GG, de Bendeicits ML, et al. Radial verus femoral approach for percutaneous coronary diagnostic and interventional procedures: systematic overview and meta-analysis of randomized trials. J Am Coll Cardiol 2004; 44: 349-56.

9. Jolly SS, Amlani S, Hamon M, et al. Radial versus femoral access for coronary angiography or intervention and the impact on major bleeding and ischemic events: a systematic review and meta-analysis of randomized trials. Am Heart J 2009; 157: 132-40.

10. Mamas MA, Fraser DG, Ratib K, et al. Minimising radial injury: prevention is better than cure. Eurointervention 2014; 10: 824-32.

11. Pancholy SB, Patel TM. Effect of duration of hemostatic compression on radial artery occlusion after transradial access. Catheter Cardiovasc Interv 2012; 79: 78-81. 